\title{
Acute myelomonocytic leukaemia presenting as xanthomatous skin eruption
}

\author{
JR O'DONNELL, P TANSEY, P CHUNG, AK BURNETT, J THOMSON, GA MCDONALD \\ From the Departments of Haematology, Pathology and Dermatology, Glasgow Royal Infirmary, Glasgow G4 \\ OSF
}

SUMMARY A case of acute myelomonocytic leukaemia (AMMOL) is reported in which skin infiltration with xanthomatous nodules was the presenting feature. The histological, including ultrastructural, appearances are described.

Skin and mucosal infiltrates are a well recognised feature of the monocytic leukaemias. We report a case of AMMOL which presented with xanthomatous skin nodules, a form of leukaemic skin infiltration not previously reported.

\section{Case report}

A 44-year-old man presented in October 1980 with multiple yellowish skin nodules on the anterior and posterior trunk and limbs (Fig. 1). The lesions were not painful or pruritic and had been present for six weeks. There were no other symptoms or significant previous complaints and no family history of disordered lipid metabolism. The lesions were absent on the palms, soles, tendon sheaths and buttocks and there was no gingival hypertrophy. There was no lymphadenopathy, hepatosplenomegaly, sternal tenderness, bruising or bleeding. Haemoglobin was $8.0 \mathrm{~g} / \mathrm{dl}$, white cell count $22.7 \times 10^{9} / 1$ and platelet count $62.0 \times 10^{9} / 1$. Blood film revealed $40 \%$ blast cells. Repeated attempts at marrow examination resulted in dry taps, but a trephine biopsy (Jamshidi needle, 11 gauge, regular adult size) from the posterior iliac crest provided material for trials from which a diagnosis of AMMOL (M4) was made, according to the criteria of the French-AmericanBritish (FAB) Cooperative Group.'

Chest $x$-ray and ECG were normal. Total cholesterol (fasting) was $2.9 \mathrm{mmol} / \mathrm{l}$ (normal range $4 \cdot 1-7 \cdot 4)$. Total triglyceride (fasting) was $0 \cdot 95 \mathrm{mmol} / 1$ (normal range $0-1 \cdot 8$ ). Lipoprotein electrophoresis was normal.

Induction chemotherapy was commenced comprising daunorubicin, cytosine arabinoside and thioguanine. After four full induction courses of this therapy bone marrow remission was achieved in December 1980. Although the xanthomatous lesions

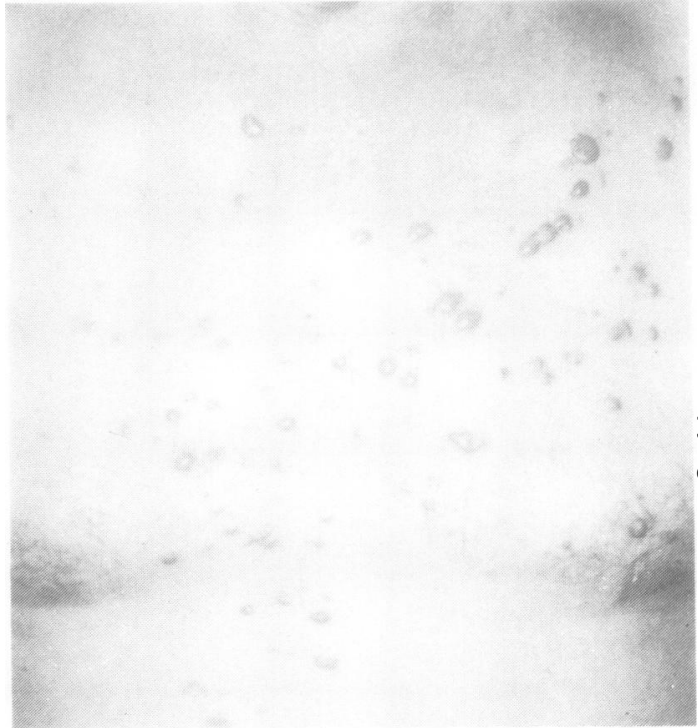

Fig. 1 Photograph of patient at presentation showing xanthomatous lesions on upper trunk.

diminished in number and size they did not entirely disappear. Repeated estimations of serum lipids revealed no abnormality. Skin infiltration again became prominent and was partly haemorrhagic (Fig. 2) after the completion of two courses of consolidation chemotherapy consisting of daunorubicin, cytosine arabinoside and thioguanine. Peripheral blood examination on this occasion exhibited blast cells of similar morphological appearance to that of the initial presentation. The patient died suddenly before further chemotherapy could be instituted. Permission for post-mortem examination was not granted.

Prior to the initiation of antileukaemic therapy, two of the skin lesions were biopsied. Biochemical analysis revealed a high concentration of triglycerides 


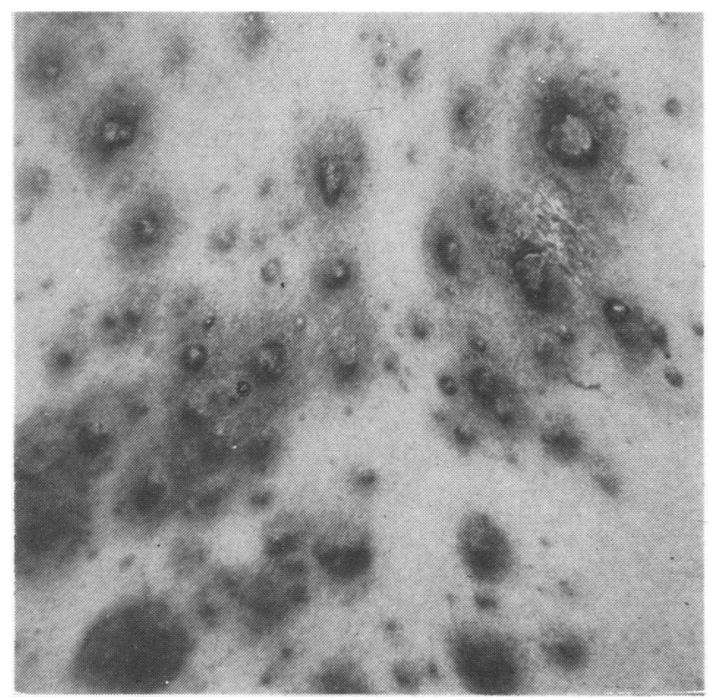

Fig. 2 Photograph of posterior trunk of patient post chemotherapy at time of final relapse of $A M M O L$. The xanthomatous lesions are partly haemorrhagic.

but only small amounts of cholesterol. Histological examination of both specimens showed a light perivascular cellular infiltrate in the upper dermis. A few plasma cells could be recognised, but it was not possible to categorise the majority of cells which exhibited nuclear pleomorphism, mitotic activity, and variable amounts of amphophilic/eosinophilic cytoplasm with occasional granules. A discrete nodule comprising a cellular aggregate infiltrating the collagen bundles of the mid and lower dermis was found in the first biopsy. The overall impression was of a xanthomatous lesion, with several unusual features. The majority of the cells had large, irregular, pale nuclei with nucleoli and abundant basophilic foamy cytoplasm (Fig. 3) resembling macrophages. Mitotic figures were prominent. A

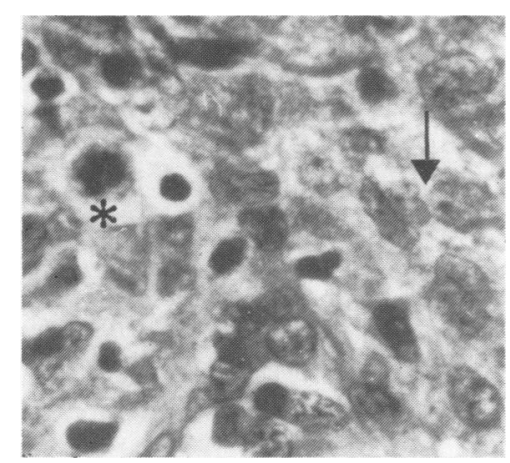

Fig. 3 Xanthoma cells,(arrow) with large nuclei, prominent nucleoli and abundant foamy cytoplasm. Mitotic figure (asterisk) Haematoxylin and eosin. $\times 240$.

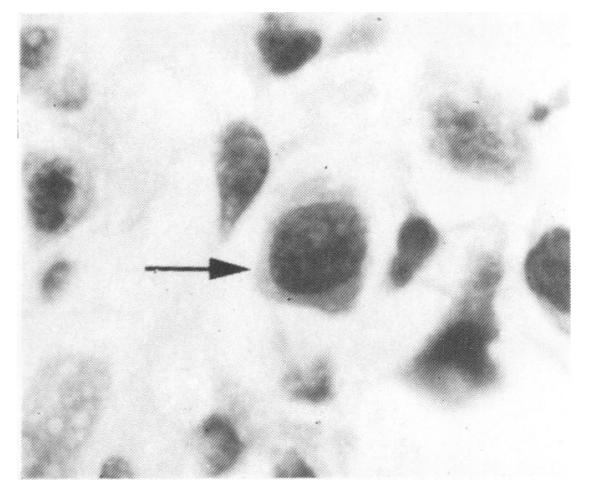

Fig. 4 The large central cell has a well defined border with pale slightly granular cytoplasm and two nucleoli, in contrast to the foamy pattern of the xanthoma cell at the bottom left. Haematoxylin and eosin. $\times 600$.

smaller number of multinucleate giant cells with similar nuclear and cytoplasmic features were present. In addition, scattered throughout the lesion, were cells with large rounded nuclei, some distinctly nucleolated and with scanty eosinophilic cytoplasm resembling myeloid precursors (Fig. 4). A number of these cells were Sudan black- and chloracetatepositive. Occasional mast cells, mature lymphocytes, mature eosinophils and eosinophil myelocytes were present.

Ultrastructural studies revealed closely packed cells with complex interdigitating surfaces, the cell membranes being in close apposition but lacking adhesion specialisations (Fig. 5). The cytoplasm was abundant and contained a number of organelles including fat vacuoles, single membrane-bound granules consistent with lysosomes, mitochondria, short profiles of rough and smooth endoplasmic reticulum and randomly disposed microfilaments (Fig. 6). The nuclei were large with a variable degree of infolding and prominent nucleoli with some segmentation interconnected by fine chromatin strands (Fig. 7). A little heterochromatin with peripheral margination was present.

\section{Discussion}

Extramedullary tissue infiltration by leukaemic cells is a well recognised and typical manifestation of the monocytic leukaemias. Such infiltrates, particularly of the skin and gingival mucosae were present in approximately one third of a recent intensively studied group of 45 patients with AMMOL or with acute monocytic leukaemia (AMOL). ${ }^{2}$ The wide variety of dermatological manifestations which may be found in the monocytic and other leukaemias is detailed in the monograph of Bluefarb. ${ }^{3}$ These include a large number of non-specific cutaneous 


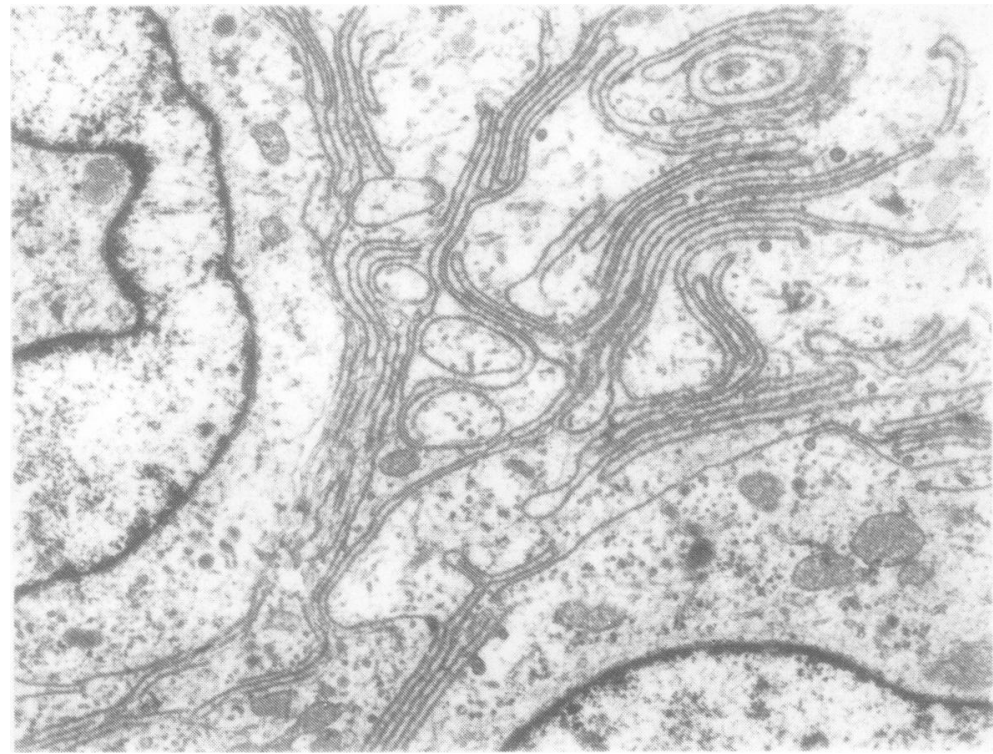

Fig. 5 Xanthoma cells showing complex interdigitation of adjacent cell membranes. $\times 9500$.

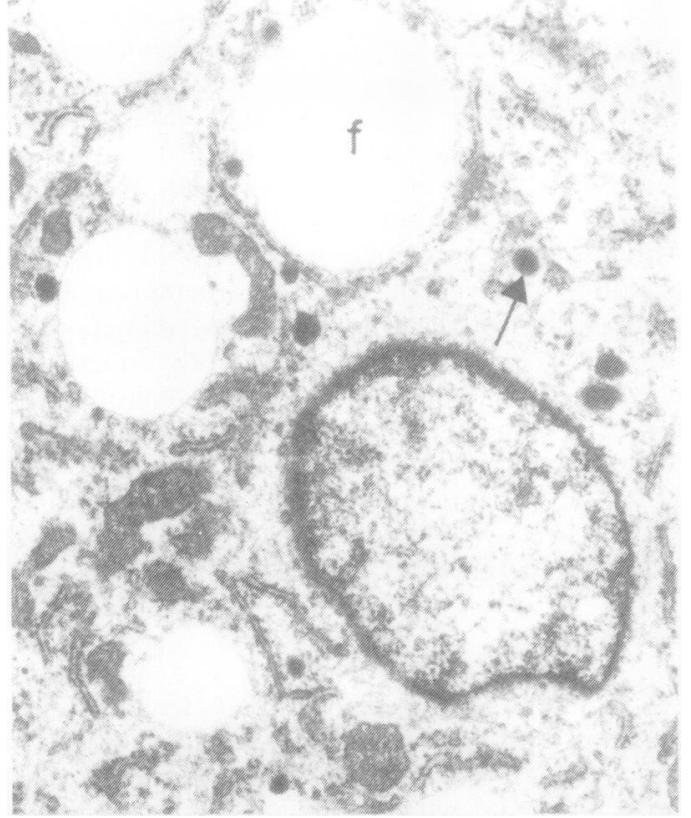

Fig. 6 Fat vacuoles $(f)$ and lysosomes (arrow) in a xanthoma cell. $\times 16000$.

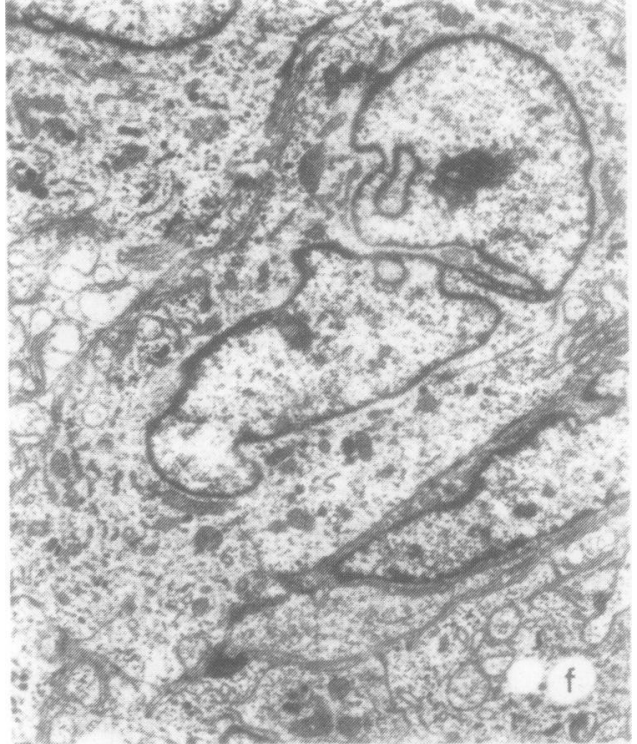

Fig. 7 Nucleus of cell from xanthomatous lesion showing nucleolation and lobulation. The two lobes are interconnected by a fine chromatin strand. The scanty heterochromatin is marginated. Cytoplasm is abundant with fat vacuoles $(f) . \times 3500$. 
features such as pruritus, exfoliative dermatitis, hyperpigmentation, erythema multiforme and bullous pemphigoid. Leukaemic lesions may take the form of solid papules or tumours macroscopically indistinguishable from infiltrates seen in the malignant lymphomas. The lesions in the monocytic leukaemias, however, tend to be large and purple or "plum" coloured and frequently ulcerate. ${ }^{4}$ As in the case we describe, skin tumours tend to spare the face. ${ }^{5}$ Skin lesions may antedate haematological expression of leukaemia by a variable period so that a wide differential diagnosis may be considered in such patients. Even at biopsy a leukaemic origin cannot definitely be assigned except in the case of chloroma (granulocytic sarcoma), the only localised tumour pathognomonic of leukaemia. ${ }^{6}$

Our case presented with xanthomatous skin lesions, a form of skin infiltration we believe has not hitherto been described. No evidence of a systemic disorder of lipid metabolism was found. A diagnosis of AMMOL was made on the basis of Romanowsky and cytochemical stains of the trails of marrow obtained at bone marrow biopsy. Auer rods were not seen but the cytochemical pattern of Sudan black positivity combined with positive alpha-napthyl acetate and naphthol-AS-D chloracetate esterases was clearly evident.

The light microscopic appearances of the xanthomatous nodules showed a degree of cellular pleomorphism and mitotic activity which suggested neoplasia rather than a diagnosis of eruptive xanthoma or infective granuloma. In addition, there was evidence of granulocytic infiltration in the presence of eosinophil myelocytes and Sudan blackand chloracetate-positive cells scattered amongst the xanthoma cells.

Electron microscopy also demonstrated an infiltrate consisting predominantly of lipid containing macrophages but was of special value in showing absence of specialised intercellular junctions, indicating a non-epithelial origin of the tumour. In occasional cells cytoplasmic electron-dense granules suggested the presence of early myeloid precursors but several other features similar to those described by Glick and Horn, ${ }^{7}$ in peripheral blood and marrow, suggested the presence of monocytes and promonocytes; the latter characterised by large indented nuclei with prominent nucleoli, numerous free ribosomes and many short segments of rough endoplasmic reticulum. O'Brien, Catovsky and Costello have shown ${ }^{8}$ that monoblasts contain a previously undescribed small lysosomal granule characterised by presence of acid phosphatase activity and lack of myeloperoxidase activity, a useful ultrastructural differentiation feature between monocytic and granulocytic precursors. At the time of relapse, the xanthomata became more prominent on our patient but it was not possible to rebiopsy the lesions of obtain fresh tissue imprints for further cytochemistry because of the patient's sudden death.

It is possible only to speculate that the leukaemic monoblasts in our patient were capable of phagocytosing lipid to a degree which led to xanthomatous nodule formation in the skin. Permission for post-mortem examination was not granted so that it is impossible to know if organs other than skin were infiltrated. The "xanthomata", although diminished in size and number, remained evident during the two-month marrow remission. It appears likely that they provided a "sanctuary" for leukaemic cells during this period.

We wish to thank Dr Mary MacGregor (Department of Pathology, Glasgow Royal Infirmary) for advice on the dermatopathology in this case.

\section{References}

' Bennet JM, Catovsky D, Daniel MT, et al. Proposal for the classification of the acute leukaemias. French-American-British (FAB) Cooperative Group. Br J Haematol 1976;33:451-8.

2 Straus DJ, Mertelsmann R, Koziner B, et al. The acute monocytic leukaemias: multidisciplinary studies in forty-five patients. Medicine 1980;59:409-25.

${ }^{3}$ Bluefarb SM. Leukaemia Cutis. Springfield Illinois: Charles C Thomas, 1960.

+ Braverman IM. Leukaemia and allied disorders in: skin signs of systemic disease. Philadelphia: Sanders, 1970:101-13.

${ }^{5}$ Gunz FW, Baikie AG. Leukemia. 3rd ed 1974. New York: Grune and Stratton, 1974:290.

- O'Donnell JR, Farrell MA. Acute myelogenous leukaemia with bilateral mammary gland involvement. J Clin Pathol 1980;33:547-51.

' Glick AD, Horn RG. Identification of promonocytes and myeloid precursors in acute leukaemia of adults: ultrastructural and cytochemical observations. Br J Haematol 1974;26:395-403.

${ }^{*} \mathrm{O}^{\prime B r i e n ~ M}$, Catovsky D, Costello D. Untrastructural cytochemistry of leukaemic cells: characterisation of the early small granules of monoblasts. Br J Haematol 1980;45:201-8.

Requests for reprints to: Dr JR O'Donnell, Department of Haematology, Glasgow Royal Infirmary, Glasgow, G4 OSF, Scotland. 